\title{
Video Article \\ Monitoring Tumor Metastases and Osteolytic Lesions with Bioluminescence and Micro CT Imaging
}

\author{
Ed Lim ${ }^{1}$, Kshitij Modi ${ }^{1}$, Anna Christensen ${ }^{1}$, Jeff Meganck ${ }^{1}$, Stephen Oldfield ${ }^{1}$, Ning Zhang ${ }^{1}$ \\ ${ }^{1}$ Imaging Biology Research and Development, Caliper Life Sciences \\ Correspondence to: Ning Zhang at Ning.Zhang@CaliperLS.Com \\ URL: https://www.jove.com/video/2775 \\ DOI: doi:10.3791/2775
}

Keywords: Medicine, Issue 50, osteolytic lesions, micro CT, tumor, bioluminescence, in vivo, imaging, IVIS, luciferase, low dose, co-registration, 3D reconstruction

Date Published: 4/14/2011

Citation: Lim, E., Modi, K., Christensen, A., Meganck, J., Oldfield, S., Zhang, N. Monitoring Tumor Metastases and Osteolytic Lesions with Bioluminescence and Micro CT Imaging. J. Vis. Exp. (50), e2775, doi:10.3791/2775 (2011).

\section{Abstract}

Following intracardiac delivery of MDA-MB-231-luc-D3H2LN cells to Nu/Nu mice, systemic metastases developed in the injected animals. Bioluminescence imaging using IVIS Spectrum was employed to monitor the distribution and development of the tumor cells following the delivery procedure including DLIT reconstruction to measure the tumor signal and its location.

Development of metastatic lesions to the bone tissues triggers osteolytic activity and lesions to tibia and femur were evaluated longitudinally using micro CT. Imaging was performed using a Quantum FX micro CT system with fast imaging and low X-ray dose. The low radiation dose allows multiple imaging sessions to be performed with a cumulative X-ray dosage far below LD50. A mouse imaging shuttle device was used to sequentially image the mice with both IVIS Spectrum and Quantum FX achieving accurate animal positioning in both the bioluminescence and CT images. The optical and CT data sets were co-registered in 3-dimentions using the Living Image 4.1 software. This multi-mode approach allows close monitoring of tumor growth and development simultaneously with osteolytic activity.

\section{Video Link}

The video component of this article can be found at https://www.jove.com/video/2775/

\section{Protocol}

Longitudinal imaging is used in pre-clinical studies to follow the progress of a disease or measure the effect of a therapeutic. In oncology, optical methods provide rigorous tools to monitor tumor growth and deliver precise quantitation of cell growth or gene expression at each time point in such a study. Anatomical changes can be measured using a high resolution technique like microCT, but for longitudinal imaging a low X-ray dose must be used to avoid biological artifacts. Optical and microCT images can be co-registered to provide a combination of functional and anatomical data ensuring that maximum information is extracted from the animal model.

\section{Cell preparation}

1. Caliper provides a range of luciferase expressing cancer cell lines for pre-clinical research in mouse models.

2. MDA-MB-231-luc-D2H2LN is a human mammary tumor cell line expressing the luciferase gene which can be used as an optical indicator of tumorgenesis in vivo. This cell line is created from a spontaneous lymph node metastasis that originated from a D3H1 mammary fat pad tumor and is known to aggressively form metastases.

3. The cells are provided as a pathogen-free frozen culture which readily grows in standard media with no need for selection markers.

4. To verify Luciferase activity before injection into the animal a $90 \%$ confluent flask is harvested by trypsinization. Luciferase activity is measured by dispensing 50,000 cells in a microtiter plate and performing serial dilution.

\section{Intracardiac injection of cells in animals}

1. Before injection, animals are anesthetized using $3 \%$ isoflurane.

2. 1-3 million cells are injected in $50 \mathrm{ul}$ volume in to the left ventricle. Cell suspension contains $150 \mathrm{ug} / \mathrm{ml} \mathrm{D}$-Luciferin to validate injection technique.

3. To confirm a correct intracardiac injection mice are imaged in the IVIS system. Mice that show BLI signal throughout the body have been injected correctly. If signal is localized the cells were not injected correctly in the heart. 


\section{BLI imaging to monitor metastases}

1. The imaging shuttle supports the mouse for data capture in both IVIS Spectrum and the QuantumFX microCT system.

2. The shuttle helps maintain the animal in the same position for imaging and incorporates fiducial signals to aid in automatic co-registration. The chamber is connected to the isofluorance vaporizer to maintain anesthesia.

3. Imaging wizard in the Living Image software 4.1 can be used to automatically select the exposure time, f-stop and binning and ensure quantitative data collection.

4. Multiple images can be acquired and compared in longitudinal studies covering seconds or months depending on the nature of the experiment.

5. Day 6 post injection of cells, BLI imaging with IVIS Spectrum shows metastases to the bone in seven out of nine mice.

\section{Longitudinal monitoring of osteolytic lesions using micro CT}

1. Mice that showed clear bone metastases visible using BLI were imaged in the Quantum FX micro CT system.

2. The Quantum FX micro CT system delivers high quality images at an X-ray dose low enough to enable true longitudinal micro CT in preclinical studies.

3. Bone degradation in both legs can be longitudinally monitored using this system in less than a minute.

4. Mice are anesthetized with $3 \%$ isoflurane and can be transferred directly from the IVIS Spectrum system.

5. Two $60 \mathrm{~mm}$ images are stitched together to capture the whole animal view.

6. A $30 \mathrm{~mm}$ FOV is ideal for imaging the knee joint in high resolution.

7. A single scan with high enough resolution to measure and quantify bone metastases takes just 18 seconds and delivers a dose of only $13 m G y$.

8. Mice were imaged up to 8 times in 36 days with the micro CT system without affecting the tumor growth or health of the animal.

9. The micro CT imaging showed that bone leisions were visible on Day 10 and progressed very rapidly, corresponding to the increase in BLI signal.

\section{3D co-registration and analysis of BLI and $\mathrm{UCT}$}

1. Once images have been captured on both the IVIS spectrum and Quantum FX micro CT system data can be co-registered into a 3D reconstruction.

2. MicroCT images are exported as DICOM files and loaded into the Living Image 4.1 3D Multi modality tool.

3. In the Living Image Software run the DLIT program to three dimensionally reconstruct the optical signal.

4. The fiducial tool in the software is used to co-register the optical BLI signal to the micro CT image.

5. Coregistered data sets can be analyzed quantitatively to monitor multiple parameters during disease progression here we are tracking growth of the metastatic tumor together with development of the osteolytic lesion in the bone.

\section{Discussion}

Small animal imaging with microCT has been applied to monitoring osteolytic lesions under pathological conditions, such as tumor development (Cowey et al. 2007; Labrinidis et al., 2009). As bioluminescence imaging of luciferase labeled tumors has been widely adopted pre-clinically, co-registration of bioluminescence and microCT images provided a better definition of anatomical location of the bioluminescence signals (Kaijzel et al. 2007). Previous study with a luciferase labeled breast tumor cell line MDA-MB-231-luc-D3H2LN showed metastases to bone and development of osteolytic lesions following intra-cardiac injection (Jenkins et al 2005; Minn et al., 2005). In this study, we explored a longitudinal study of tumor metastases and osteolytic activity with combinatory optical and low-radiation-dose microCT imaging with the systemic MDAMB-231-luc-D3H2LN metastasis model. We performed sequential imaging with IVIS Spectrum and Quantum FX microCT systems and achieved bioluminescence and CT co-registration with our newly developed Living Image 4.1 software (Heng et al., 2010). Bioluminescence imaging with IVIS provides non-invasive detection of the tumor cells with high sensitivity, allowing the tumors to be detected at early stage. Imaging with Quantum FX allows accurate 3D reconstruction of the bone lesions and detailed anatomical information. Quantum FX system offers low radiation dose imaging, which enables longitudinal monitoring of disease progression. Correlation of bone lesions and tumor metastases was depicted in the co-registered image, and the advantage of multimodality tracking of lesion development longitudinally was clearly demonstrated.

\section{Disclosures}

The authors are employees of Caliper Life Sciences who make the instrument featured in this article.

\section{References}

1. Jenkins, D.E., Hornig, Y.S., Oei, Y., Dusich, J., Purchio, T. Bioluminescent Human Breast Cancer Cell Lines that Permit Rapid and Sensitive in vivo Detection of Mammary Tumors and Multiple Metastases in Immune Deficient Mice. Breast Cancer Research. 7, R444-R454 (2005).

2. Cowey, S., Szafran, A. A., Kappes, J., Zinn, K.R., Siegal, G.P., Desmond, R. A., Kim, H., Evans, L., Hardy, R. W. Breast Cancer Metastasis to Bone: Evaluation of Bioluminescent Imaging and MicroSPECT/CT for Detecting Bone Metastasis in Immunodeficient Mice. Clinical and Experimental Metastasis. 24 (5), 389-401 (2007).

3. Labrinidis, A., Hay, S., Liapis, V., Ponomarev, V., Findlay, D.M., Evdokiou, A. Zoledronic Acid Inhibits Both the Osteolytic and Osteoblastic Components of Osteosarcoma Lesions in a Mouse Model. Clinical Cancer Research. 15(10) (2009). 
4. Kaijzel, E.L., van der Pluijm, G., Lowik, C.W.G.M. Whole-Body Optical Imaging in Animal Models to Assess Cancer Development and Progression. Clinical Cancer Research. 13 (12), 3490-3497 (2007).

5. Minn, A.J., Kang, Y., Serganova, I., Gupta, G.P., Giri, D.D., Doubrovin, M., Ponomarev, V., Gerald, W.L., Blasberg, R., Massague, J. Distinct Organ-Specific Metastatic Potential of Individual Breast Cancer Cells and Primary Tumors. The Journal of Clinical Investigation. 115 (1), (2005).

6. Xu, H, Christensen A., Kuo C., Nilson D., Lim E., Whalen J., Kim, J.B., Zhang, N., Singh, R., Oldfield, S., Troy, T., Rice, B. Co-registration of in vivo Optical Tomography and Micro-CT for Longitudinal Studies. WMIC. \#0332B, Kyoto (2010). 